\title{
An Objective Measure of Stimulus-Evoked Pain
}

\author{
John L. K. Kramer, Jenny Haefeli, and Catherine R. Jutzeler \\ Spinal Cord Injury Center, University Hospital Balgrist, University of Zurich, CH 8008 Zurich, Switzerland \\ Review of Zhang et al.
}

For most clinical assessments, reporting perception of a painful event has relied on subjective ratings [e.g., the visual analog scale (VAS)]. A more quantifiable and objective method is desirable, preferably one that is directly related to the physiological processing of nociceptive stimuli. An ideal noninvasive method for this purpose is to use laser stimulation, which activates purely nociceptive $\mathrm{A} \delta / \mathrm{C}$-fibers in the periphery, and record laser-evoked potentials (LEPs) generated in the cortex. Brain mapping studies have established a positive relationship between the intensity of pain reported to laser stimuli and LEP amplitude, as well as disclosed a network of cortical areas that are involved in processing laser stimuli.

Whether LEPs directly represent the experience of pain or a more generalized response of heightened attention or arousal to afferent stimuli has been the topic of considerable debate (Truini et al., 2004, 2007; Iannetti et al., 2008; Mouraux and Iannetti, 2008, 2009; Baumgärtner and Treede, 2009; Lee et al., 2009; Wang et al., 2010; Mouraux et al., 2011). Several lines of evidence have recently emerged that suggest LEPs are, at least in part, attributable to multimodal cortical processing of saliency to afferent stimuli. First, non-nociceptive sensory stimuli

Received July 4, 2012; revised July 24, 2012; accepted July 25, 2012.

John Kramer is supported by the International Foundation for Research in Paraplegia—Zurich. We are thankful to Drs. Armin Curt (Spinal Cord Injury Center, University Hospital Balgrist) and John Steeves (ICORD, University of British Columbia) for reviewing this manuscript.

Correspondence should be addressed to Dr. John L. K. Kramer, Postdoctoral Fellow, Spinal Cord Injury Center, University of Zurich, University Hospital Balgrist, Forchstrasse 340, CH 8008 Zurich, Switzerland. E-mail: jkramer@paralab.balgrist.ch.

DOI:10.1523/JNEUROSCI.3175-12.2012

Copyright $\odot 2012$ the authors $\quad 0270-6474 / 12 / 3212981-02 \$ 15.00 / 0$ (e.g., auditory and visual stimuli) evoke cortical potentials that are difficult to distinguish from prominent LEPs (Mouraux and Iannetti, 2009). Second, following nonnociceptive multimodal stimuli, hemodynamic responses have been observed within the same areas activated by nociceptive laser stimulation (Mouraux et al., 2011).

To uncouple the contributions of stimulation saliency and perceived intensity, Iannetti et al. (2008) used a stimulation protocol in which three consecutive laser stimuli of the same energy were delivered at $1 \mathrm{~Hz}$. Triplets were applied at four different intensities to produce varying perception of pain. The authors proposed that the first of the three stimuli remains salient due to a long intertrain interval ( $20 \mathrm{~s}$ ), but it increases expectancy and reduces the novelty for subsequent stimuli (Iannetti et al., 2008). The net effect of stimulus repetition is a reduction in LEP amplitude of the averaged second and third stimuli relative to that of the first. The modulation of LEP amplitude by stimulus repetition is observed without corresponding changes in perceived rating of intensity. Importantly, amplitudes generated by the second and third stimuli were reduced for both the earlier contralateral negative component $(\mathrm{N} 1$, considered a more direct measure of nociceptive input), and for the vertex potential (N2P2).

Iannetti et al. (2008) suggested that stimulus saliency, not neural refractoriness ["first come, first serve" hypothesis, proposed by Truini et al. $(2004,2007)]$, accounted for the reduced amplitude of LEPs generated at short interpulse intervals. Indeed, neural refractoriness cannot account for changes in LEP amplitude because subjective perception to laser stimuli does not change over the course of the three delivered pulses (Iannetti et al., 2008). Furthermore, the arrival of a second, unexpected stimulus generates an LEP of equal amplitude, even when delivered at relatively short interpulse intervals (Mouraux et al., 2004; Wang et al., 2010).

Ultimately, the goal of triplet paradigm is to identify key-evoked potential parameters that are less affected by stimulus energy and repetition (i.e., salient features) and more strongly correlated with the subject's perception of pain intensity. According to a recent study (Zhang et al., 2012), this is where gamma band oscillations (GBOs) might play a pivotal role in pain research.

Zhang and colleagues (2012) reexamined data from the original triplet laser stimulation study (Iannetti et al., 2008) with a focus on GBOs. The relationship between GBOs, stimulus repetition, and stimulus intensity is perhaps best illustrated in the left panel of Zhang et al.'s (2012) Figure 3. While a relationship emerges between rating of perceived stimulus intensity, LEPs, and GBOs (i.e., change in magnitude of responses as stimuli are rated as being more painful), only GBOs are unaffected by stimulus repetition. That is, GBOs do not appreciably change from one stimulus to the next, and thus, correspond with the rating of perceived intensity to a given stimuli. In the subsequent figures by Zhang et al. (2012), this point is illustrated and substantiated by statistical analysis revealing that the intensity of pain (i.e., subjective pain expe- 
rience), but not stimulus repetition or stimulus energy, resulted in significant changes in GBO magnitude.

While Zhang and colleagues (2012) were not the first to identify the relationship between GBOs and pain intensity, the reanalysis of the triplet laser stimulation data controls for the potential confound of stimulus saliency. Given the marked effect of saliency on LEPs, it was important to test whether GBOs behave similarly. In contrast to LEP amplitude, laser-induced GBOs appear to represent a more direct measure of pain perception. Whether the emerging characteristics of noxious GBOs differ from innocuous GBOs (e.g., those produced by nonaversive visual or acoustic stimuli) remains an important question to address in future studies.

One potential challenge of acquiring laser-evoked GBOs will be to isolate oscillations strictly related to the nociceptive stimulus from artifacts generated by microsaccades. To account for eye movements, Zhang and colleagues (2012) used independent component analysis to remove ocular artifacts. However, to further minimize the contribution of ocular artifacts, future experiments that aim to study pain-evoked GBOs should consider recently developed guidelines (Schwartzman and Kranczioch, 2011). These guidelines include the use of longer duration stimuli and measuring GBOs at ocular recording electrodes.

If GBOs induced by laser stimulation can be independently and prospectively validated as a strong correlate of perceived pain intensity, the clinical implications are far reaching. In particular, laser-induced GBOs could be useful to more accurately diagnose patients with complex sensory deficits, such as neuropathic pain. Indeed, this is an area where current LEP parameters have met with limited success (Hatem et al., 2010). In the context of clinical trials, GBOs may be useful to objectively monitor and track pain relief due to a therapeutic intervention (i.e., measuring changes in GBOs preintervention and postintervention, along with patient-reported VAS score). Monitoring continuous GBOs may also improve the objective assessment of pain. Such a technique could be equally or potentially more meaningful than event-related GBOs to diagnose spontaneous or idiopathic pain.

\section{References}

Baumgärtner U, Treede RD (2009) Are there nociceptive-specific brain potentials? J Neurophysiol 102:3073-3074; author reply 30753076. CrossRef Medline

Hatem SM, Attal N, Ducreux D, Gautron M, Parker F, Plaghki L, Bouhassira D (2010) Clinical, functional and structural determinants of central pain in syringomyelia. Brain 133:3409-3422. CrossRef Medline

Iannetti GD, Hughes NP, Lee MC, Mouraux A (2008) Determinants of laser-evoked EEG responses: pain perception or stimulus saliency? J Neurophysiol 100:815-828. CrossRef Medline

Lee MC, Mouraux A, Iannetti GD (2009) Characterizing the cortical activity through which pain emerges from nociception. J Neurosci 29: 7909-7916. CrossRef Medline

Mouraux A, Iannetti GD (2008) A review of the evidence against the "first come first served" hypothesis. Comment on Truini et al. [Pain
2007;131:43-47]. Pain 136:219-221; author reply 222-223. CrossRef Medline

Mouraux A, Iannetti GD (2009) Nociceptive laser-evoked brain potentials do not reflect nociceptive-specific neural activity. J Neurophysiol 101:3258-3269. CrossRef Medline

Mouraux A, Guérit JM, Plaghki L (2004) Refractoriness cannot explain why C-fiber laserevoked brain potentials are recorded only if concomitant a[delta]-fiber activation is avoided. Pain 112:16-26. CrossRef Medline

Mouraux A, Diukova A, Lee MC, Wise RG, Iannetti GD (2011) A multisensory investigation of the functional significance of the "pain matrix”. Neuroimage 54:2237-2249. CrossRef Medline

Schwartzman DJ, Kranczioch C (2011) In the blink of an eye: the contribution of microsaccadic activity to the induced gamma band response. Int J Psychophysiol 79:73-82. CrossRef Medline

Truini A, Rossi P, Galeotti F, Romaniello A, Virtuoso M, De Lena C, Leandri M, Cruccu G (2004) Excitability of the a[delta] nociceptive pathways as assessed by the recovery cycle of laser evoked potentials in humans. Exp Brain Res 155:120-123. CrossRef Medline

Truini A, Galeotti F, Cruccu G, Garcia-Larrea L (2007) Inhibition of cortical responses to a[delta] inputs by a preceding C-related response: testing the "first come, first served" hypothesis of cortical laser evoked potentials. Pain 131:341-347. CrossRef Medline

Wang AL, Mouraux A, Liang M, Iannetti GD (2010) Stimulus novelty, and not neural refractoriness, explains the repetition suppression of laser-evoked potentials. J Neurophysiol 104: 2116-2124. CrossRef Medline

Zhang ZG, Hu L, Hung YS, Mouraux A, Iannetti GD (2012) Gamma-band oscillations in the primary somatosensory cortex: a direct and obligatory correlate of subjective pain intensity. J Neurosci 32:7429-7438. CrossRef Medline 